\title{
A Flight Strategy for Intelligent Aerial Vehicles Learned from Dragonfly
}

\author{
Zheng $\mathrm{Hu}$ and Xinyan Deng \\ University of Delaware \\ U.S.A.
}

\section{Introduction}

Dragonfly is one of the most maneuverable insects and one of the oldest flying species on earth. It is important for human beings to study their flight techniques if we intend to make an insect-like Micro Aerial Vehicle, because their flight performance far exceeds other insects. They can hover, cruise up to $54 \mathrm{~km} / \mathrm{h}$, turn $180^{\circ}$ in three wing beats, fly sideways, glide, and even fly backwards (Alexander, 1984; Appleton, 1974; Whitehouse, 1941). They intercept prey in the air with amazing speed and accuracy. Their thorax are equipped with wing muscles which accounts for $24 \%$ (Aeshna) of its body weight, compared to $13 \%$ of those of the honey bees (Appleton, 1974). Most dragonflies change their wing motion kinematics for different flight modes such as hovering, cruising and turning. Among these kinematic parameters, the most interesting one is the phase difference $(\gamma)$ between forewing and hindwing. It is defined as the phase angle by which the hindwing leads the forewing. When hovering, dragonflies employ $180^{\circ}$ phase difference (anti-phase) (Alexander, 1984; Norberg, 1975; Rüppell, 1989), while 54 $100^{\circ}$ are used for forward flight (Azuma and Watanabe, 1988; Wang et al., 2003). When accelerating or performing aggressive maneuvers, they use $0^{\circ}$ (inphase) phase difference (Alexander, 1984; Rüppell, 1989; Thomas et al., 2004). Of various phase differences, $270^{\circ}$ is rarely observed in dragonfly flight.

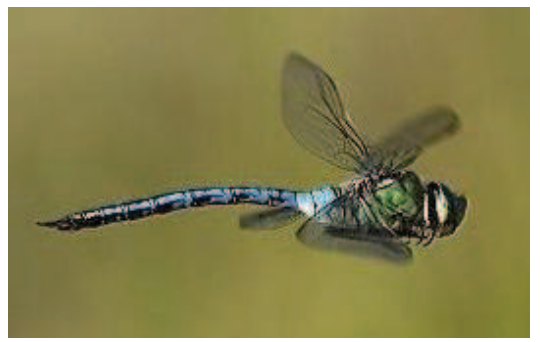

Figure 1. Phase difference in dragonfly

The fact that flapping in-phase $\left(0^{\circ}\right)$ appears in situations requiring large acceleration suggests that in-phase might produce higher forces (Alexander, 1984; Rüppell, 1989). The film sequences made by Alexander (Alexander, 1984) showed that in-phase is employed during take-off and sharp turning. It was also found that in a rising flight of a dragonfly, lift was increased during downstroke and drag was increased during upstroke when flying in- 
phase (Azuma et al., 1985). The conclusion was derived by using the momentum theory and the blade element theory, combined with a numerical method modified from the local circulation method.

However, it was argued that as two wings in tandem are brought closer together, the lift force produced by each wing is reduced (Alexander, 1984). Therefore, forewing and hindwing flapping in-phase would produce less lift because they are closer together than when beating anti-phase. Alexander believed that the reason of dragonflies using in-phase flight may be due to physiological reason as well as the preference of peak forces enhancement at the cost of the mean forces reduction (Alexander, 1984).

Counterstroking $\left(180^{\circ}\right.$ or anti-phase) produces uniform flight, whereas flight produced by parallel stroking $\left(0^{\circ}\right)$ is irregular (Rüppell, 1989). This is because inequalities in the aerodynamic effects of the upstroke and downstroke can be compensated to some extent in countertroking. As one pair of wing's upstroke with a steep angle of attack generates strong thrust, the other pair's downstroke with a small angle of attack mainly generates lift. Therefore the net thrust and lift production remains relatively constant during flight due to the alternating force generation on two wings (Rüppell, 1989).

In a recent computational study, (Wang and Russell, 2007) calculated dragonfly's aerodynamic force and power as a function of forewing-hindwing phase difference. They found that anti-phase flapping consumes nearly minimal power while generating sufficient force to balance body weight, and that in-phase motion provides an additional force to accelerate (Wang and Russell, 2007). Furthermore, they proposed an analogy to explain the results by analyzing a model of two cylinders moving in parallel next to each other.

Other computational studies include (Wang and Sun, 2005) and (Huang and Sun, 2007), where they calculated the aerodynamic effects of forewing-hindwing interactions of a specific dragonfly (Aeshna juncea) in hover and slow forward flight. They showed that the interaction is detrimental to force generation in almost all cases. At hovering with $\gamma=180^{\circ}$, the reduction is $8 \sim 15 \%$, compared with the force without interaction. The force on hindwing is greatly influenced by the forewing at $\gamma=180 \sim 360^{\circ}$, with the lift coefficient decreased by $20 \sim 60 \%$. Furthermore, they proposed a mechanism to explain the effect of forewing on hindwing force reduction: the forewing in each of its downstroke produces a downward "jet" behind it; when the hindwing lags the forewing, it moves into the jet and its effective angle of attack is reduced, resulting in a decrease in its aerodynamic force.

Previous computational studies include (Lan, 1979), where the unsteady quasi-vortex-lattice method was applied to the study of dragonfly aerodynamics, and the results showed that dragonfly can produce high thrust with high efficiency if hindwing leads the forewing by $90^{\circ}$, and that hindwing was able to extract wake energy from the forewing under this condition.

Direct force measurements on tethered dragonflies showed that peak lift increases from approximately 2 to 6.3 times body weight when the animal decreases the phase difference between both flapping wings (Reavis and Luttges, 1988). But maybe this enhancement on peak lift may due to overlap of peak lifts on forewing and hindwing, not necessarily due to wing-wing interaction.

Experimental investigations of the aerodynamic effect of wing-wing interaction was previously performed in (Maybury and Lehmann, 2004), where a pair of robotic wings were vertically stacked to simulate dragonfly hovering flight with horizontal stroke plane. They found that the lift production of the forewing remains approximately constant, while hind 
wing lift production is reduced to some extent and its maximum value occurs at a phase difference $\gamma=90^{\circ}$. They attributed the wing-wing interaction to two reasons: LEV destruction and local flow condition (Maybury and Lehmann, 2004). Their results explained the hovering behavior of dragonflies using horizontal stroke plane (Sympetrum Sanguineum), while many other dragonfly species employ a $20 \sim 70^{\circ}$ inclined stroke plane (Alexander, 1984; Norberg, 1975; Rüppell, 1989) and employ an aerodynamic mechanism of "drag based lift generation" (Wang, 2004), which is quite different from that for horizontal stroke plane flight (Wang and Russell, 2007). In this study, we investigate the wing-wing interaction and the underlying mechanism for the inclined stroke plane species.

The effect of the forewing-hindwing interactions in dragonflies has been investigated with some computational and experimental studies, but conclusions are still limited and quite varying. In this study, we constructed a pair of robotic dragonfly wings to investigate the aerodynamic effect of wing-wing interactions in both hovering and forward flight. This apparatus enables us to study inclined stroke plane species such as Aeshna Juncea with an inclined stroke plane $60^{\circ}$ and varying forward speed. The wing bases are of close proximity to mimic the dragonfly wings. We systematically vary the different phase between the forewing and hindwing to find out why dragonflies apply certain phase differences rather than others in certain flight modes. The conclusion from this research would be valuable for designing a dragonfly-inspired M.A.V (Micro Air Vehicle).

\section{A robotic dragonfly model}

The Micro Robotics Lab of University of Delaware constructed a pair of dynamically-scaled robotic wing models (flappers) to replicate dragonfly wing motion and measure the instantaneous aerodynamic forces when flapping in a tank filled with mineral oil (Fig. 2).
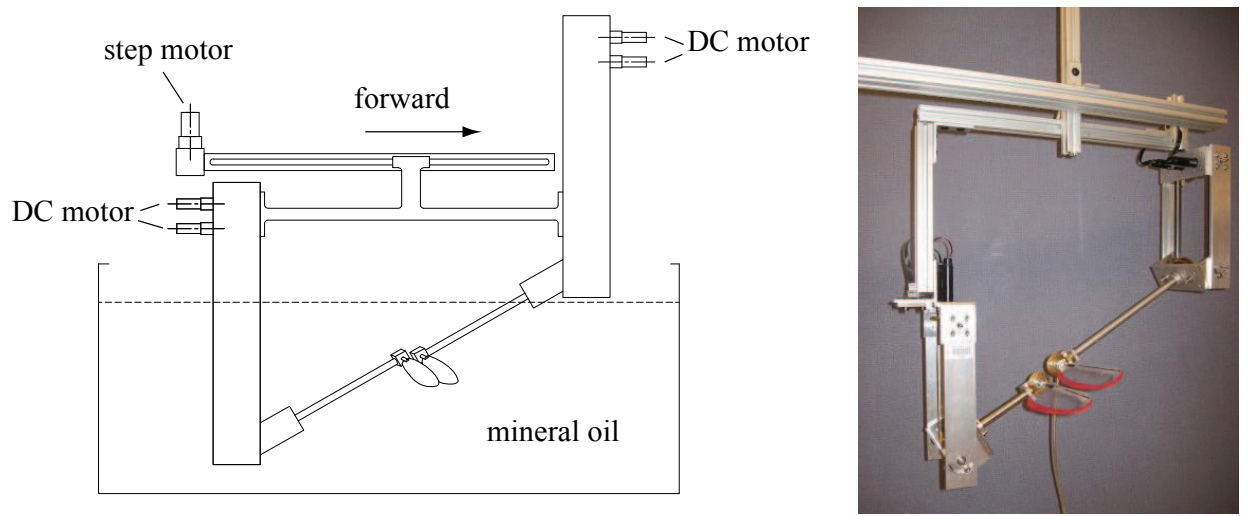

Figure 2. Sketch and image of the experimental setup

For each flapper, a bevel-geared robotic wrist was designed to generate motions of two degrees of freedom (translation and rotation). A set of bevel gears transmits the motion from coaxially driven shafts to the wing holder thus enabling wing translation and rotation (Fig. $3)$. The two wings are placed with a very close proximity like those of a true dragonfly. In addition, the two flappers are mounted on a linear stage driven by a step motor to achieve forward motion together. 
The drive shafts were powered by $16 \mathrm{~mm}, 0.3 \mathrm{Nm}$ torque DC brush motors (Maxon, Sachseln, Switzerland) equipped with gear heads to reduce speed and magnetic encoders to provide kinematic feedback to ensure motion fidelity. The motors were driven along kinematic patterns provided by a custom MATLAB (Mathworks, Natick, MA) Simulink program with WinCon software (Quanser Consulting, Ontario, Canada). This software provided commands to the real-time control and data acquisition board (Quanser Consulting, Ontario, Canada) communicating with the hardware. We used ProportionalIntegral-Derivative (PID) controllers to run the motors with precision of $0.1^{\circ}$. Motion commands from the computer were amplified by analog amplifier units (Advanced Motion Control) which directly controlled the input current received by the motor.

Wings were made from Mylar plastic film with a thickness of $0.25 \mathrm{~cm}$ which behaves as a rigid wing in our experiments. A carbon fiber rod was glued on the plastic film to serve as the leading edge. The end of the carbon fiber rod was affixed on to the force sensor. The wings have identical geometry as the dragonfly wings but are four times larger with a length of $19 \mathrm{~cm}$ for forewing and $18.5 \mathrm{~cm}$ for hindwing. The wing length is calculated as the distance from wing tip to the flapping axis.

The wings along with part of the device were immersed into a tank $(46 \mathrm{~cm} \times 41 \mathrm{~cm} \times 152 \mathrm{~cm})$ filled with mineral oil (Kinematic viscosity $=3.4 \mathrm{cSt}$ at $20 \mathrm{oC}$, density $=830 \mathrm{~kg} / \mathrm{m} 3$ ). This overall set-up enabled us to run the wings along a pre-determined dragonfly kinematics while simultaneously measuring the forces on the forewing and the hindwing respectively (Fig. 2).

\section{Flight pattern of a typical dragonfly}

High-speed photos of the dragonfly (Aeshna juncea) in hovering flight were taken by Norberg (Norberg, 1975). The body is held almost horizontal, and the wing stroke plane is tilted $60^{\circ}$ relative to the horizontal line. For both forewing and hindwing, the chord is almost horizontal during the downstroke and is close to being vertical during the upstroke (Fig. 4); the stroke frequency is $36 \mathrm{~Hz}$, the stroke amplitude is $60^{\circ}$; the translational angle is from $35^{\circ}$ above the horizontal to $25^{\circ}$ below for forewing, and is from $45^{\circ}$ above to $15^{\circ}$ below for hindwing; the hindwing leads the forewing in phase by $180^{\circ}$. The mass of the insect is $754 \mathrm{mg}$; forewing length is $4.74 \mathrm{~cm}$; hindwing length is $4.60 \mathrm{~cm}$; the mean chord lengths of the forewing and hindwing are $0.81 \mathrm{~cm}$ and $1.12 \mathrm{~cm}$, respectively; the moment of inertial of wing-mass with respect to the fulcrum is $4.54 \mathrm{~g} \mathrm{~cm}^{-2}$ for the forewing and $3.77 \mathrm{~g} \mathrm{~cm}^{-2}$ for the hindwing (Norberg, 1972).

Due to the low frame rate of the camera used at that time $(80 \mathrm{~Hz})$, Norberg's data does not consist of a detailed continuous trajectory of the wing kinematics. Azuma instead, (Azuma et al., 1985) successfully filmed a slow climbing flight of a dragonfly (Sympetrum Frequens) with a high speed camera (873 frames per second). He showed that the flapping trajectory can be well represented by a sinusoidal function and the rotation trajectory can be represented by a third harmonic function. Since the two species (Aeshna juncea and Sympetrum frequens) share similar values for many kinematic parameters such as translational amplitude, rotational amplitude and stroke plane angle (Azuma et al., 1985; Norberg, 1975), here we assume they also employ similar wing motion trajectories. This assumption of kinematic trajectories can be reasonably applied to other species with highly inclined stroke planes without affecting the main results of this study. We developed a pair 
of wing kinematic trajectories by matching those for Sympetrum Frequens in (Azuma et al., 1985), as shown in Fig. 5.
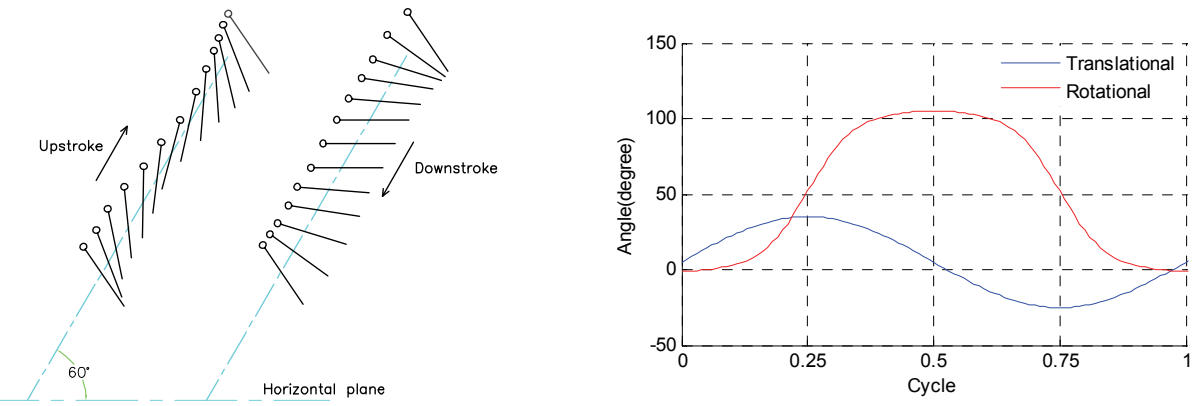

Figure 4. (left) Sketches of the dragonfly wing kineamtics during upstroke and downstroke based on Aeshna juncea data (Norberg, 1975). Side views with circles denote the leading edge. Both forewing and hindwing apply; (right) Wing kinematics developed by matching those for Sympetrum Frequens from (Azuma et al., 1985) and (Norberg, 1975). Translational angle (blue) and rotation angle (red). This is for forewing only; the kinematics for hindwing is almost the same except a $10^{\circ}$ offset for translational angle

\section{Force measurements $\&$ calculations}

For each wing, we measured the instantaneous lift and thrust forces and calculated the average forces. The forces as well as torques on the moving wings can be measured by a sixcomponent force sensor (ATI NANO-17, Apex, NC), with a range of $\pm 12 \mathrm{~N}$ for force and \pm 0.5 $\mathrm{Nm}$ for torque along three orthogonal axes. Using appropriate trigonometric conversions, these force measurements were then converted to lift and thrust forces in the earth coordinates. Note that in this study, lift force is defined as the vertical component of the aerodynamics force on the wing and thrust force is the horizontal component in global coordinate frame.

The magnitude of aerodynamic forces acting on an actual dragonfly, $F_{f l y}$, is related to those measured in the robotic model, $F_{\text {robot }}$, according to the following scaling rule (Fry et al., 2005):

$$
F_{f l y}=F_{\text {robot }} \cdot \frac{\rho_{\text {air }}}{\rho_{\text {oil }}} \cdot\left(\frac{n_{f l y}}{n_{\text {robot }}}\right)^{2} \cdot\left(\frac{r_{f l y}}{r_{\text {robot }}}\right)^{2} \cdot \frac{S_{f l y}}{S_{\text {robot }}} \cdot \frac{\hat{r}_{2 f l y}^{2}}{\hat{r}_{2 \text { robot }}^{2}}
$$

where $\rho$ is fluid density, $\mathrm{n}$ is stroke frequency, $\mathrm{r}$ is wing length, $\mathrm{S}$ is wing area, and $r_{2}$ is the normalized second moment of wing area. $\hat{r}_{2 f l y}$ and $\hat{r}_{2 \text { robot }}^{2}$ differ slightly, due to a small null length at the base of the robotic wing required to accommodate the force sensor. In this study, lift force is defined as the vertical component of the aerodynamics force on the wing; thrust force is the horizontal component. Lift coefficient and thrust coefficient are respectively defined in the following way, similar to (Wang and Sun, 2005): 


$$
\begin{aligned}
& C_{l}=L /\left[0.5 \rho U^{2}\left(S_{f}+S_{h}\right)\right] \\
& C_{t}=T /\left[0.5 \rho U^{2}\left(S_{f}+S_{h}\right)\right]
\end{aligned}
$$

$L$ and $T$ denote the total lift and the total thrust. The reference velocity is calculated to be $U=2 \Phi n r_{2}=2.1 \mathrm{~ms}^{-1} ; S_{f}$ and $S_{h}$ are the areas of forewing and hindwing, respectively.

Accordingly, we define lift coefficient and thrust coefficient for a single wing. For example, for forewing:

$$
\begin{aligned}
& C_{l, f}=L_{f} /\left[0.5 \rho U^{2}\left(S_{f}+S_{h}\right)\right] \\
& C_{t, f}=T_{f} /\left[0.5 \rho U^{2}\left(S_{f}+S_{h}\right)\right]
\end{aligned}
$$

The total lift coefficient and total thrust coefficient are as follows:

$$
\begin{gathered}
C_{l}=C_{l, f}+C_{l, h} \\
C_{t}=C_{t, f}+C_{t, h}
\end{gathered}
$$

The lift coefficient on a dragonfly should be 2 times $C_{l}$, since it has two forewings and two hindwings.

\section{Aerodynamic force for anti-phase hovering flight}

We first replay the hovering kinematics of dragonfly on the robotic flappers and measured lift and thrust forces on the both wings. Here the forewing and hindwing are anti-phase $\left(180^{\circ}\right)$, which is most commonly observed in dragonfly hovering flight. The results show an average lift force of $0.0711 \mathrm{~N}$ on the forewing and $0.082 \mathrm{~N}$ on the hindwing during one beat cycle. The average thrust forces are $0.001 \mathrm{~N}$ on the forewing and $0.003 \mathrm{~N}$ on the hindwing.

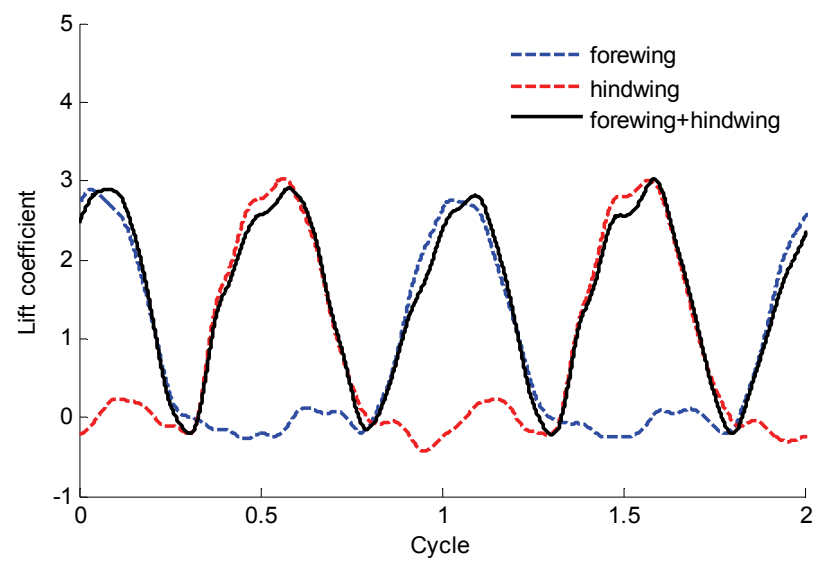

Figure 6. Time trace of lift force coefficients generated when hovering with $\gamma=180^{\circ}$ 
We then apply (1) to scale the lift force back to those of a true dragonfly. The resulted average lift force is $187 \mathrm{mg}$ on a forewing and $216 \mathrm{mg}$ on a hindwing. The total average lift on a four-wing dragonfly is therefore $(187+216)^{*} 2=806(\mathrm{mg})$. This result is comparable to the $754 \mathrm{mg}$ body mass measured in (Norberg, 1972). On the other hand, the total average thrust force is almost zero when compared with the average lift force, which follows the hovering condition that the thrust should be zero.

The time traces of instantaneous lift forces are shown in Fig.6. Both forewing and hindwing produce lift peaks during downstrokes. Because the flapping of forewing and hindwing are anti-phase, they generate lift force alternatively during one wingstroke. The average lift force on hindwing is 1.15 times that on forewing, while the area of hindwing is 1.32 times that of forewing. This inconsistency is probably caused by the fact that, compared to forewing, hindwing has more area distributed at positions close to the body. It was proposed that for dragonfly flight, the hindwing acts as power wing which provide more lift force while the forewing is the steering wing (Azuma and Watanabe, 1988; Wang et al., 2003). As we can see, the total lift force is generated alternatively by the forewing and hindwing during one wingbeat. By doing so the insect is able to hover with regular forces which reduce the vibration of the body.

\section{Effect of phase difference in hovering flight}

We measured the instantaneous forces with the same kinematics of hovering but systematically vary the phase differences from $0^{\circ}$ to $360^{\circ}$ in steps of $30^{\circ}$. The average lift coefficients of both wings according to varying phase difference are plotted in Fig. 7.

Fig. 7 shows the averaged force coefficients in hover. As the phase difference tends to $0^{\circ}$, forces tend to be higher, and reach their maximum on $0^{\circ}$. The forces get lower values when $\gamma$ is around $180^{\circ}$. An interesting point is that not all values are below the single wing force, that is, the wing-wing interaction is not always detrimental to force generation. We can conclude here, in range $330^{\circ}$ to $30^{\circ}$, the wing-wing interaction enhances the total lift force by up to $6 \%$; in range $150^{\circ}$ to $180^{\circ}$, interaction decreases the total lift force by up to $9 \%$. The thrust forces are much smaller than the lift forces, which is reasonable for hovering flight.
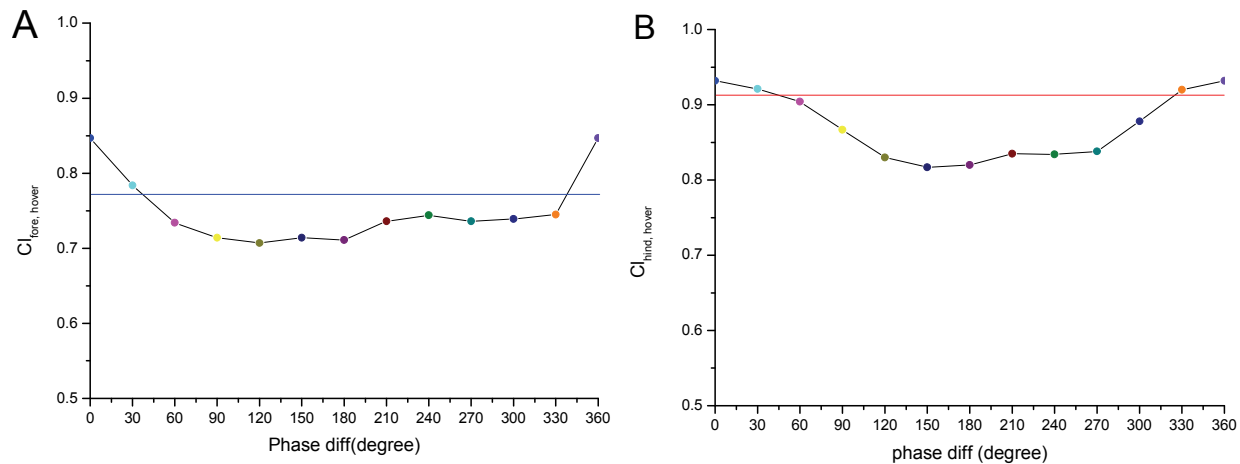

Figure 7. Force coefficients results for hovering flight test. A) Average lift coefficients on forewing; B) average lift coefficients on hindwing; C) total average lift coefficients. The straight lines in each plot indicate the force results without interaction 
This part of study supplies a direct proof for the statement that in-phase flight generates larger aerodynamic forces than anti-phase flight, and also larger than the case without wingwing interaction. This may explain the behavior that dragonfly flies in-phase in case of accelerating or maneuverings that calls for a high force generation.

In order to find out the possible reason that dragonfly uses anti-phase style for hovering mode, we need to compare the time traces of total lift forces generated by $\gamma=0^{\circ}$ with those generated by $\gamma=180^{\circ}$ (Fig. 8). It was noted that anti-phase produces uniform flight, whereas flight produced by in-phase stroking is irregular (Rüppell, 1989). Fig. 8 shows the instantaneous lift force coefficients comparison between in-phase and anti-phase flight. As we can see, in-phase brings larger irregularity in the aerodynamic forces than anti-phase does. Observing from the time trace curve for in-phase hovering, there exists a $1 / 2$ cycle period when lift force is closed to zero, while in another $1 / 2$ cycle the peak value is two times of the peak value for anti-phase flight, because forewing and hindwing peak overlap. This irregularity of instantaneous forces increases the body vibration when hovering, while for anti-phase flight the inequality can be compensated to some extent by evenly distributing the peak forces of forewing and hindwing on the whole cycle. Besides minimizing the force irregularities and keeping body posture stable, anti-phase flight can also save energy that might be lost in body vibration (Wang and Russell, 2007). Moreover, Usherwood and Lehmann showed that dragonfly can also save aerodynamic power during anti-phase hovering (Usherwood and Lehmann, 2008). Thus, it is reasonable that dragonflies would rather lose $15 \%$ force production for improving flight stability and vibration suppression as well as power efficiency.

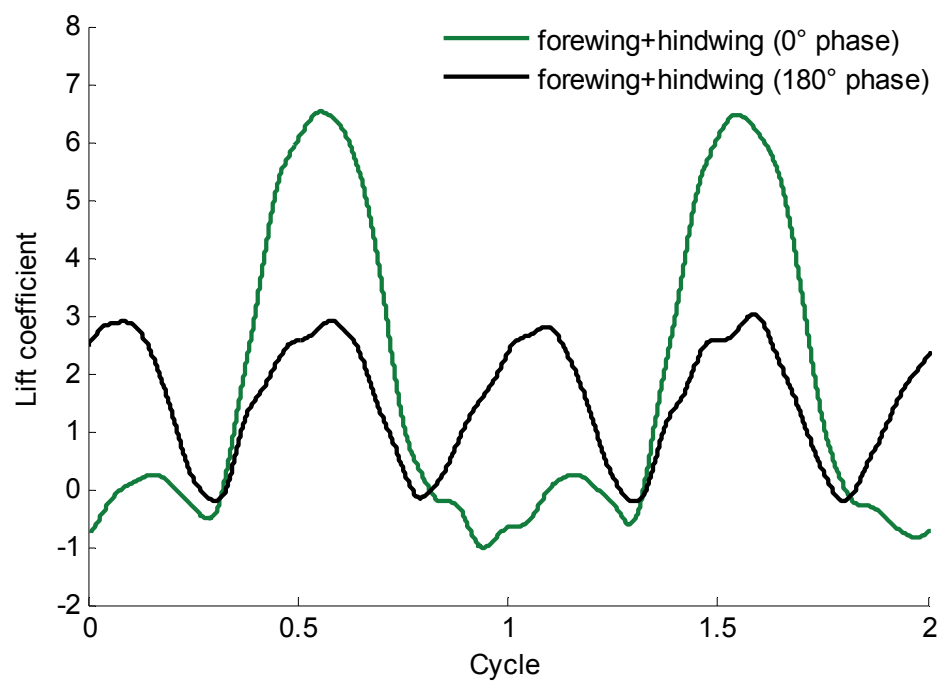

Figure 8. Comparison between total lift forces generated when hovering with $\gamma=0^{\circ}$ and $\gamma=$ $180^{\circ}$ respectively 


\section{Effect of phase difference in forward flight}

Similarly to the hovering case, we tested the wing aerodynamics by varying phase difference systematically in steps of $30^{\circ}$. The average lift coefficients and average thrust coefficients of both wings are plotted in Fig. 9.

As seen in Fig. 9, during forward flight, interaction patterns for forewing and hindwing differ a lot. Note that the interaction always enhances the forewing's force generation no matter how much $\gamma$ is, while the hindwing always loses force production because of the interaction. Lift on forewing is enhanced by at most $23 \%$ when flapping in-phase and at least $4 \%$ when phase difference falls into $120^{\circ} \sim 330^{\circ}$; Lift on hindwing reaches maximum on $60^{\circ}$ and minimum on $270^{\circ}$. Hindwing is subjected to a severe loss on force production up to $38 \%$ due to the interaction with forewing. The total force on the two wings does not lose so much force as the hindwing does, due to the considerable enhancement on forewing lift.

Fig. 10 compares the time traces of hindwing lift among the cases of single hindwing, $\gamma=90^{\circ}$ and $\gamma=270^{\circ}$. We note that the lift in case of $\gamma=270^{\circ}$ was considerably reduced when compared with other cases, and the reduction mainly occurred when hindwing was in the midway of downstroke. This is reasonable because a large portion of lift is produce during this period, since wings reach the highest flapping speed and largest angle of attack around the midway of downstroke.
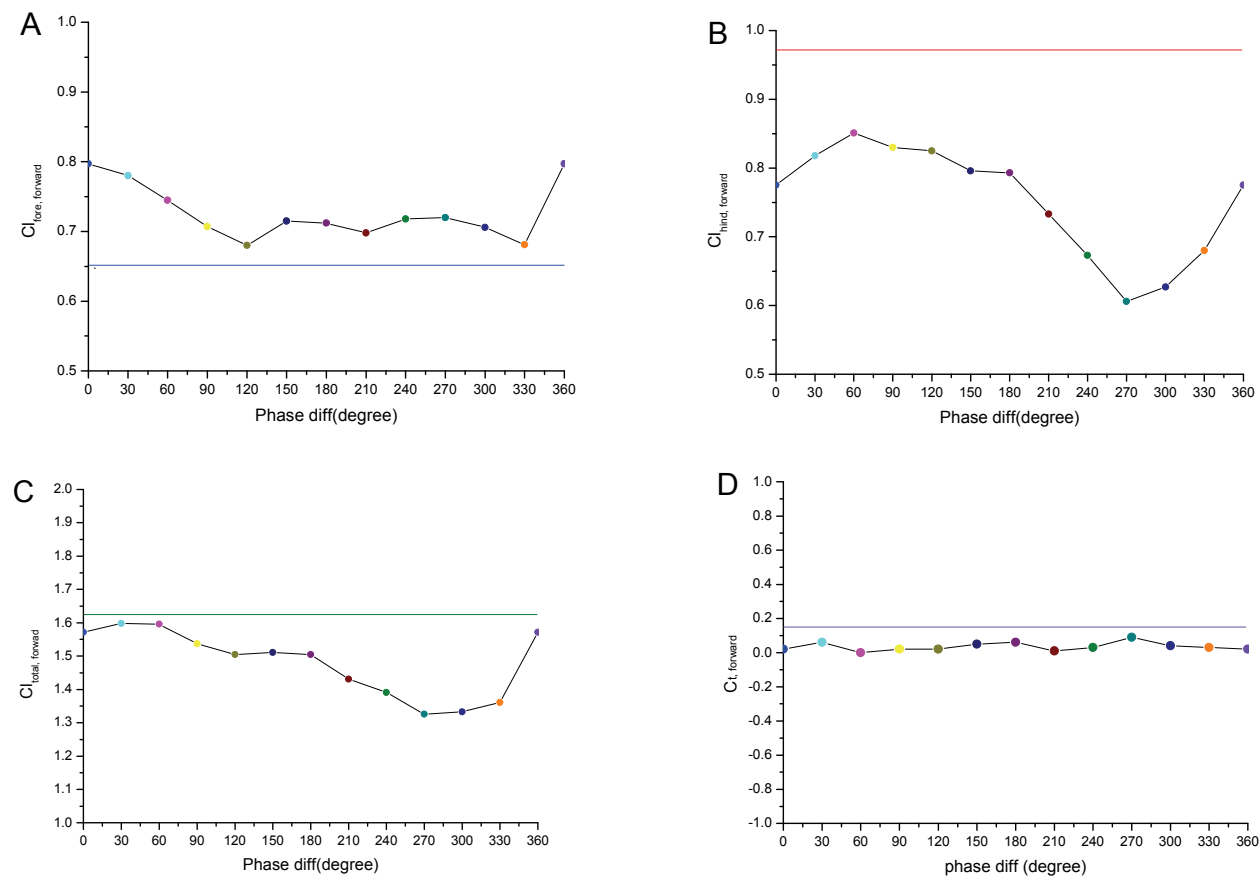

Figure 9. Force coefficients results for forward flight test. A) Average lift coefficients on forewing; B) average lift coefficients on hindwing; C) total average lift coefficients; D) total average thrust coefficients. The straight lines in each plot indicate the force results without interaction 
One may explain the reduction in the following way: forewing generates a strong downwash when it finishes a downstroke. If $\gamma=270^{\circ}$, that is, forewing leads hindwing by $1 / 4$ cycle, then hindwing is just in the midway of downstroke as forewing finishes downstroke and generates a downwash. In the mean time, because of forward flight, hindwing enters into the downwash area and its lift production decreases due to the downwash effect.

Now we can distinguish the case $\gamma=90^{\circ}$ and the case $\gamma=270^{\circ}$ : the former one can offer dragonfly an $18 \%$ higher force than the latter one. On the other hand, $270^{\circ}$ offers similar vibration and stability properties as $90^{\circ}$. This may explain why dragonfly never favors the $270^{\circ}$ phase difference.

The above results agree qualitatively with the CFD results from (Wang and Sun, 2005) and (Huang and Sun, 2007) to some extent. Their conclusion is that the forewing is only slightly influenced by the wing-wing interaction, but the hindwing lift is greatly reduced by $20 \sim 60 \%$ during forward flight with a $180^{\circ} \sim 360^{\circ}$ phase difference, compared with that of a single hindwing. In our results, furthermore, there are obvious lift enhancements on the forewing. For thrust force measurement, (Warkentin and DeLaurier, 2007) conducted a systematic series of wind-tunnel tests on an ornithopter configuration consisting of two sets of symmetrically flapping wings, located one behind the other in tandem. It was discovered that the tandem arrangement can increase thrust for certain relative phase differences and longitudinal spacing between the wing sets. In particular, close spacing on the order of one chord length is generally best, and phase differences of approximately $-50^{\circ}$ to $50^{\circ}$ give the highest thrusts and propulsive efficiencies. Nevertheless, this conclusion does not apply for the dragonfly flight. Instead, the thrust plot above indicates a drop on thrust force caused by interaction, no matter what the phase difference is. This would be reasonable if we notice that the space between forewing and hindwing of dragonfly is much smaller that the one chord spacing between the ornithopter wing sets.

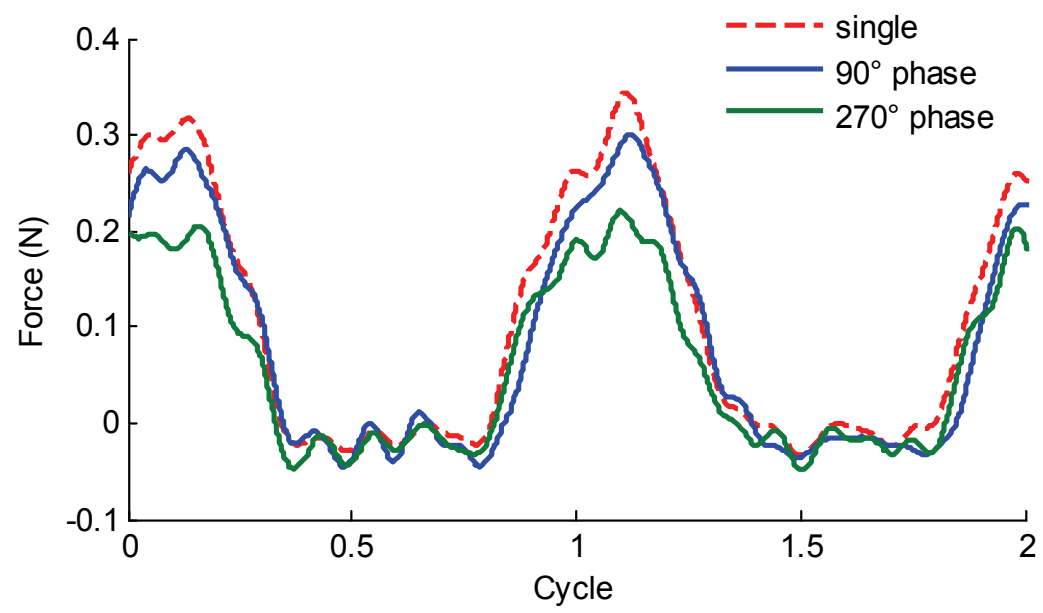

Figure 10. Comparisons of instantaneous hindwing lift in forward flight among the cases of single hindwing, $\gamma=90^{\circ}$ and $\gamma=270^{\circ}$. Note: the vertical axis indicates the forces generated on the flapper experiment and are unscaled 


\section{Dragonfly-inspired M.A.V}

At last we introduce several dragonfly robots we have developed in our lab. Fig. 11 shows the latest designs. This model has two pairs of wings driven by a double crank rocker mechanism.

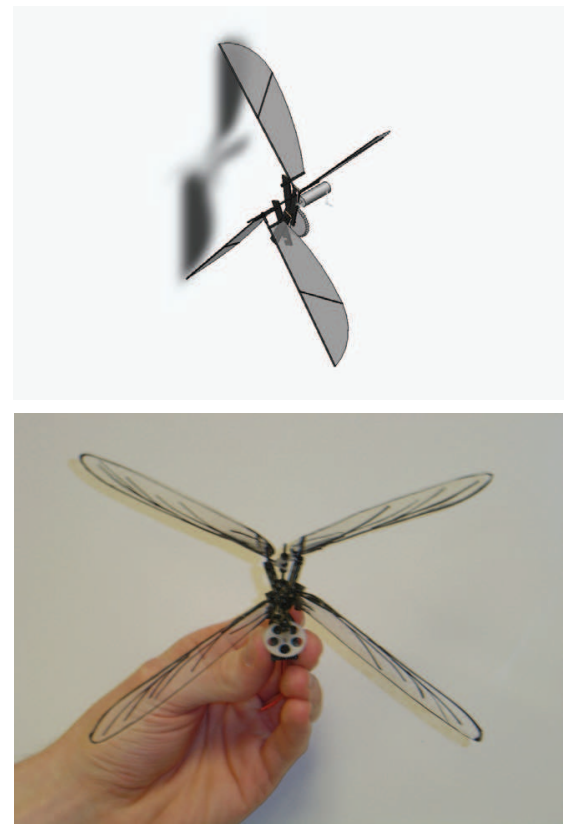

Figure 11. Dragonfly M.A.V prototype

The current prototype weighs $4 \mathrm{~g}$ including battery and electronics. The following table shows some specifications.

\begin{tabular}{|l|l|l|}
\hline Motor & Battery \& Control & Gear \\
\hline Torque: $44 \mathrm{mNm}$ & $4 \mathrm{~V}$ & Maximize Torque \\
\hline Power: $176 \mathrm{~mW}$ & $0.4 \mathrm{~A}$ & Ratio: $1: 7$ \\
\hline 16k rpm & Infrared Chip & Precision Molded \\
\hline Weight: $1.4 \mathrm{~g}$ & Weight:1.1+1.3g & Weight:0.23g \\
\hline
\end{tabular}

Table 1. Dragonfly prototype specifications

This prototype was able to generate $28 \mathrm{~Hz}$ flapping frequency with four carbon fiber leading edges only and $9 \mathrm{~Hz}$ after adding (gluing) the polymer wing onto two leading edges. With all four wings the frequency reduced to $7 \mathrm{~Hz}$. The total weight is 7 grams and the total length is 2 inches. 
In order to test different wing performance and property, we have developed a mechanical wing tester. It can be used to study the fatigue cycles of each wing developed and can be also used to visualize the wing kinematics by a high speed camera. Fig.12 shows the wing tester and a sample dragonfly wing made of carbon fiber and polymers. Fig.13 shows the camera images of test wing and Fig.14 shows the frequency plot of different robotic wings under investigation.
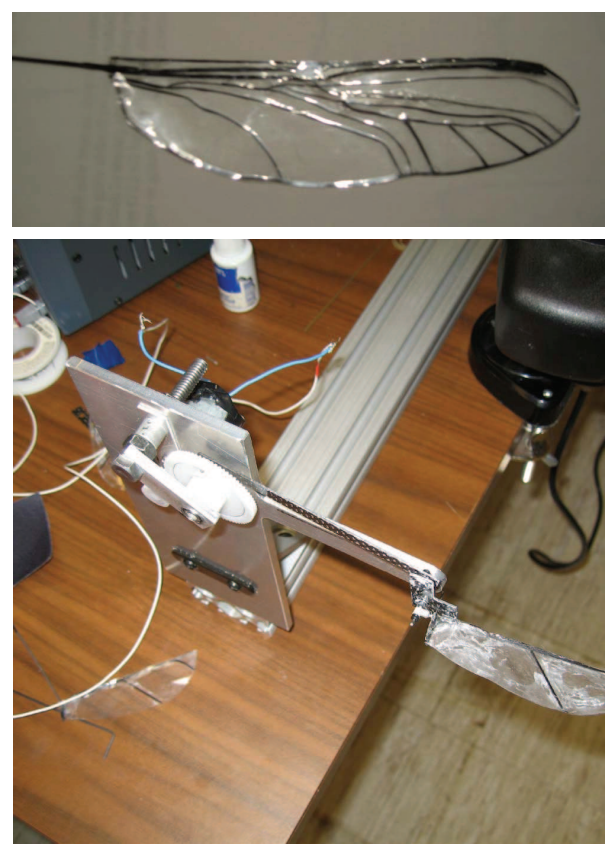

Figure 12. Sample test wing and wing tester

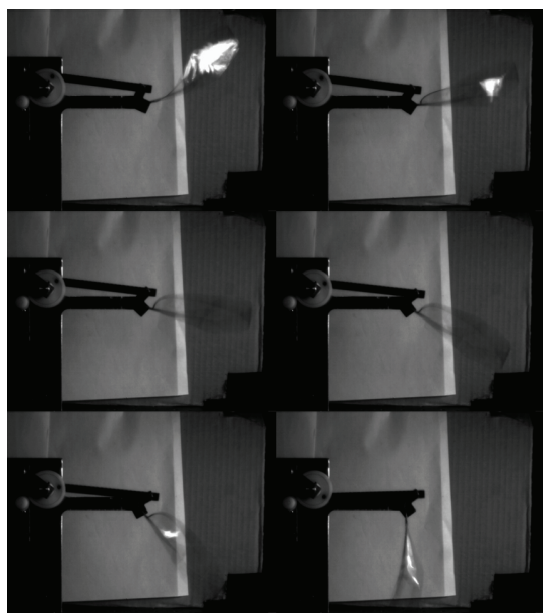

Figure 13. Dragonfly shaped wing flapping at $20 \mathrm{~Hz}$ 
The average size of the wings is bigger compared to real insects under our investigation. Veins are added for added rigidity to generate higher force. With reduced length and size, the frequency becomes higher. This process requires much trial and error as simple differences in wing structure can tear it apart at high frequencies. Dragonflies have a lot of these characteristics naturally build into their wings. Further tests will be aimed to finding out what makes their wings so robust yet so delicate under high frequencies.

\section{Conclusion and the future work}

The study described here investigates the effect of forewing-hindwing interactions during hovering and forward flight of dragonfly. Overall, wing-wing interaction is detrimental to total lift force generation. Hindwing lift was significantly reduced in forward flight due to the downwash from forewing. In-phase flight generates higher lift than other phase differences, while $270^{\circ}$ phase difference generates the lowest lift. In hovering, dragonflies use anti-phase flight which generates a regular lift force for stability and vibration reduction purposes. A prototype for dragonfly-inspired M.A.V has been shown.

Many jobs need to be done in the future aiming to build up a sophisticated M.A.V. The mechanical design should be further improved to achieve a higher flapping frequency and better efficiency, in order to minimize the weight and maximize the lift force. How to make phase difference controllable on a four-wing M.A.V is also challenging the engineers.

\section{References}

Alexander, D. E. (1984). Unusual phase relationships between the forewings and hindwings in flying dragonflies. J Exp Biol 109, 379-383.

Appleton, F. M. (1974). Dragonflies and flight. Nature Canada 3(3), 25-29.

Azuma, A., Azuma, S., Watanabe, I. and Furuta, T. (1985). Flight mechanics of a dragonfly. J Exp Biol 116, 79-107.

Azuma, A. and Watanabe, T. (1988). Flight performance of a dragonfly. J Exp Biol 137, 221252.

Fry, S. N., Sayaman, R. and Dickinson, M. H. (2005). The aerodynamics of hovering flight in Drosophila. J Exp Biol 208, 2303-18.

Huang, H. and Sun, M. (2007). Dragonfly forewing-hindwing interaction at various flight speeds and wing phasing. AIAA J. 45, 508-511.

Lan, C. E. (1979). The unsteady quasi-vortex-lattice method with applications to animal propulsion. J. Fluid Mech. 93, 747-765.

Maybury, W. J. and Lehmann, F. O. (2004). The fluid dynamics of flight control by kinematic phase lag variation between two robotic insect wings. J Exp Biol 207, 4707-26.

Norberg, R. A. (1972). The pterostigma of insect wings and inertial regulator of wing pitch. J. Comp. Physiol 81, 9-22.

Norberg, R. A. (1975). Hovering flight of the dragonfly: Aeschna juncea L., kinematics and aerodynamics. Swimming and Flying in Nature 2, 763-780.

Reavis, M. A. and Luttges, M. W. (1988). Aerodynamic forces produced by a dragonfly. AIAA J. 88-0330, 1-13.

Rüppell, G. (1989). Kinematic analysis of symmetrical flight manoeuvres of odonata. J Exp Biol 144, 13-42. 
Thomas, A. L., Taylor, G. K., Srygley, R. B., Nudds, R. L. and Bomphrey, R. J. (2004). Dragonfly flight: free-flight and tethered flow visualizations reveal a diverse array of unsteady lift-generating mechanisms, controlled primarily via angle of attack. J Exp Biol 207, 4299-323.

Usherwood, J. R. and Lehmann, F. O. (2008). Phasing of dragonfly wings can improve aerodynamic efficiency by removing swirl. $J R$ Soc Interface.

Wang, H., Zeng, L., Liu, H. and Yin, C. (2003). Measuring wing kinematics, flight trajectory and body attitude during forward flight and turning maneuvers in dragonflies. $J$ Exp Biol 206, 745-57.

Wang, J. K. and Sun, M. (2005). A computational study of the aerodynamics and forewinghindwing interaction of a model dragonfly in forward flight. J Exp Biol 208, 3785804.

Wang, Z. J. (2004). The role of drag in insect hovering. J Exp Biol 207, 4147-55.

Wang, Z. J. and Russell, D. (2007). Effect of forewing and hindwing interactions on aerodynamic forces and power in hovering dragonfly flight. Physical Review Letters 99.

Warkentin, J. and DeLaurier, J. (2007). Experimental aerodynamic study of tandem flapping membrane wings. AIAA J. 44, 1653-1661.

Whitehouse, F. C. (1941). British Columbia dragonflies (Odonata), with notes on distribution and habits. The American Midland Naturalist 26(3), 488. 


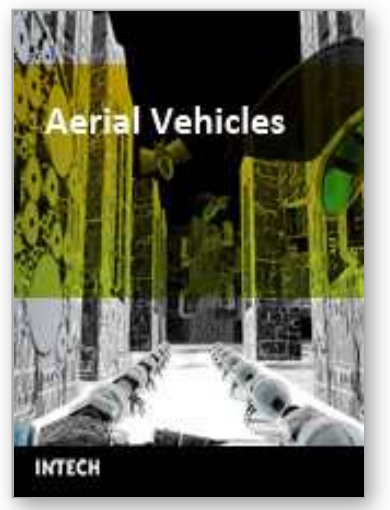

\author{
Aerial Vehicles \\ Edited by Thanh Mung Lam
}

ISBN 978-953-7619-41-1

Hard cover, 320 pages

Publisher InTech

Published online 01, January, 2009

Published in print edition January, 2009

This book contains 35 chapters written by experts in developing techniques for making aerial vehicles more intelligent, more reliable, more flexible in use, and safer in operation. It will also serve as an inspiration for further improvement of the design and application of aeral vehicles. The advanced techniques and research described here may also be applicable to other high-tech areas such as robotics, avionics, vetronics, and space.

\title{
How to reference
}

In order to correctly reference this scholarly work, feel free to copy and paste the following:

Zheng Hu and Xinyan Deng (2009). A Flight Strategy for Intelligent Aerial Vehicles Learned from Dragonfly, Aerial Vehicles, Thanh Mung Lam (Ed.), ISBN: 978-953-7619-41-1, InTech, Available from: http://www.intechopen.com/books/aerial_vehicles/a_flight_strategy_for_intelligent_aerial_vehicles_learned_fro m_dragonfly

\section{INTECH}

open science | open minds

\section{InTech Europe}

University Campus STeP Ri

Slavka Krautzeka 83/A

51000 Rijeka, Croatia

Phone: +385 (51) 770447

Fax: +385 (51) 686166

www.intechopen.com

\section{InTech China}

Unit 405, Office Block, Hotel Equatorial Shanghai

No.65, Yan An Road (West), Shanghai, 200040, China

中国上海市延安西路65号上海国际贵都大饭店办公楼405单元

Phone: +86-21-62489820

Fax: +86-21-62489821 
(C) 2009 The Author(s). Licensee IntechOpen. This chapter is distributed under the terms of the Creative Commons Attribution-NonCommercialShareAlike-3.0 License, which permits use, distribution and reproduction for non-commercial purposes, provided the original is properly cited and derivative works building on this content are distributed under the same license. 